\title{
BMJ Open Association of fatty liver index with risk of incident type 2 diabetes by metabolic syndrome status in an Eastern Finland male cohort: a prospective study
}

\author{
Olubunmi Olujimisola Olubamwo, ${ }^{1}$ Jyrki K Virtanen, ${ }^{2}$ Jussi Pihlajamaki, ${ }^{3,4}$ \\ Tomi-Pekka Tuomainen ${ }^{2}$
}

To cite: Olubamwo 00, Virtanen JK, Pihlajamaki J, et al. Association of fatty liver index with risk of incident type 2 diabetes by metabolic syndrome status in an Eastern Finland male cohort: a prospective study. BMJ Open 2019;9:e026949. doi:10.1136/ bmjopen-2018-026949

- Prepublication history and additional material for this paper are available online. To view please visit the journal (http:// dx.doi.org/10.1136/bmjopen2018-026949).

Received 28 September 2018 Revised 30 May 2019 Accepted 31 May 2019

\section{Check for updates}

(c) Author(s) (or their employer(s)) 2019. Re-use permitted under CC BY-NC. No commercial re-use. See rights and permissions. Published by BMJ.

${ }^{1}$ Public Health and Clinical Nutrition, Ita-Suomen yliopisto Terveystieteiden tiedekunta, Kuopio, Finland

${ }^{2}$ Public Health and Clinical Nutrition, Ita-Suomen yliopisto Kansanterveystiede ja kliininen ravitsemustiede, Kuopio, Finland ${ }^{3}$ Public Health and Clinical Nutrition, University of Eastern Finland School of Medicine, Kuopio, Finland

${ }^{4}$ Clinical Nutrition and Obesity Center, Kuopio University Hospital, Kuopio, Finland

Correspondence to Dr Olubunmi

Olujimisola Olubamwo; olubunmi.olubamwo@uef.fi

\section{ABSTRACT}

Objective Fatty liver disease (FLD) is increasingly recognised as a predictor of cardiometabolic risk. Our objective was to examine if metabolic syndrome (MS) status affects the association of FLD with incident type 2 diabetes (T2D) in middle-aged men.

Design Prospective epidemiological study.

Setting University affiliated research centre in Kuopio, Eastern Finland.

Participants Our subjects were 1792 Finnish men without diabetes at baseline in the KuopiolschaemicHeart Disease Risk Factor Study cohort.

Outcome measure Using fatty liver index (FLI), the association of baseline FLD with incident T2D was analysed in multivariable-adjusted Cox regression models, considering their MS statuses. The main models were adjusted for constitutional factors, lifestyle factors, biomarkers of inflammation and for high ( $F L I \geq 60$ ) versus low $(\mathrm{FLI}<30)$ FLI categories.

Results During a mean follow-up of 19 years, 375 incident cases of T2D were recorded. In the full model, the HR (HR $(95 \% \mathrm{Cl})$ ) for T2D was 3.68 (2.80 to 4.82). The association was attenuated, but maintained, with further adjustment for metabolic factors. When MS status was adjusted for in place of metabolic factors, the HRs $(95 \%$ Cls) were 2.63 (1.92 to 3.59 ) for $\mathrm{FLI} \geq 60$ and 1.77 (1.35 to 2.31) for MS. In MS-stratified analysis, FLI predicted T2D only among persons without MS. In unstratified analysis with subjects categorised by FLI-MS, persons with $\mathrm{FLI} \geq 60$ without MS had increased risk for T2D ( $H R=3.19$ (2.26 to 4.52)) compared with persons with $\mathrm{FLI}<30$ without MS. Persons with $\mathrm{FLI}<30$ and $\mathrm{MS}$ had greater risk $(\mathrm{HR}=4.31$ (2.15 to 8.61)) and persons with both FLI $\geq 60$ and MS had the greatest risk ( $\mathrm{HR}=4.66$ (3.42 to 6.35$))$.

Conclusion Generally, FLD (FLI $\geq 60$ ) predicts T2D. It specifically predicted T2D among men without MS but not among men with MS, for whom MS alone already increases the risk. Both FLI and MS can complement each other in screening and surveillance for persons with increased T2D risk.

\section{INTRODUCTION/BACKGROUND}

There is increasing recognition of the fact that fatty liver disease (FLD) is the most common cause of chronic liver disease worldwide. Also
Strengths and limitations of this study

- The study is population based, and the design is prospective, with long follow-up.

- We adjusted for a range of constitutional factors, lifestyle factors and biomarkers keeping in cognizance the components of both major exposure variables to avoid overadjustment.

- The study population comprised of men only.

- Fatty liver index used as a surrogate of fatty liver does not detect progression of fatty liver disease.

- The statuses of the men with respect to viral hepatitis were not established at baseline, although viral hepatitis has remained low in the Finnish population.

known as hepatic steatosis, FLD is associated with increased risk of cardiovascular disease (CVD) and type 2 diabetes (T2D). The prevalence has been observed to steadily rise, although this varies in different populations. Recent estimates suggest a global prevalence of $25 \%$ among adults, but the highest prevalence occurs in the Middle East and South America, while the lowest prevalence is in Africa. ${ }^{1}$ The prevalence is estimated to be $24 \%$ in Europe and more than $30 \%$ in developed countries. ${ }^{1}$ Approximately one-third of patients with FLD progress to steatohepatitis with fibrosis, which can thereafter progress to cirrhosis, liver failure and hepatocellular carcinoma. ${ }^{1}$ FLD is intimately linked with metabolic diseases, including T2D, and it can be considered a predictor of metabolic diseases, even in the non-obese population. ${ }^{2}$

While FLD is an acknowledged public health problem, there is growing interest in FLD as a predictor of incident T2D. ${ }^{3} \mathrm{~A}$ number of epidemiological studies suggest that non-alcoholic fatty liver disease (NAFLD), diagnosed using either liver enzymes or ultrasound scan, is associated with an increase in T2D incidence. ${ }^{45}$ 
Liver biopsy is the gold standard for characterising liver histology in patients with fatty liver. The procedure is expensive and carries some morbidity and very rare mortality risks. ${ }^{6}$ The fatty liver index (FLI), an algorithm comprising body mass index (BMI), waist circumference, gamma-glutamyl transferase (GGT) and triglyceride concentrations. It was developed by Bedogni et al to predict the presence of FLD. The algorithm has been widely validated and has gained increased acceptance. ${ }^{78}$ There have been reports of an association of high FLI (FLD) with incident T2D. ${ }^{9}$ However, with FLD being intimately linked with metabolic diseases, it is uncertain whether the predictive ability of FLD is independent of presence of established metabolic syndrome (MS), a known potent predictor of T2D.

Therefore, using FLI as a surrogate for FLD, we examined whether MS status affects the association of FLD, with incident T2D in middle-aged men.

\section{METHODS}

\section{Study population}

Our study population comprised participants in the Kuopio Ischaemic Heart Disease Risk Factor Study (KIHD). The KIHD study is a prospective population-based study. It was designed to investigate risk factors for CVDs and related outcomes, in middle-aged and ageing men, from Eastern Finland. The original study population consisted of an age-stratified sample of 2682 men. These were enrolled at baseline between March 1984 and December 1989. The men were 42, 48, 54 or 60 years of age at baseline. ${ }^{11}$

\section{Data collection}

Data were collected using self-administered questionnaires, interviews, physical examinations and various blood tests that aimed to elucidate physiological and biochemical parameters. ${ }^{12} 13$ The self-administered questionnaire was used to collect data on medical history, including history of T2D, metabolic diseases, liver disease and so on, medication history, family history of diabetes and family history of CVD. ${ }^{12}$ Data on lifestyle, including physical activity, history of smoking habit, history of alcohol consumption and diet, were also collected. ${ }^{14}$ Categorisation of alcohol consumption was done according to standard guidelines by the National Institute of Alcohol Abuse and Alcoholism ${ }^{15}$ and Dietary Guidelines for Americans $2010^{16}$ as already published. ${ }^{17}$

A family history of CVD or diabetes was defined as positive if the father, mother, sister or brother of the subject had a history of CVD or diabetes. ${ }^{12}$ A subject was defined as a smoker if he had ever smoked on a regular basis and had smoked cigarettes, cigars or pipe within the previous 30 days. Dietary intakes including fruit, berry and vegetable consumption were assessed with a 4-day food recording. ${ }^{18}$

Physical examinations included anthropometric indices, vital signs and physiological measurements. All measurements were made following standard protocols. Waist circumference was calculated as the mean of waist circumferences taken at maximal inspiration and maximal expiration. BMI was computed as the ratio of weight in $\mathrm{kg}$ to the square of height in metres $\left(\mathrm{kg} / \mathrm{m}^{2}\right)$. Blood pressure was taken as the mean of measurements taken in the supine, standing and sitting position with 5 min intervals. ${ }^{19}$

\section{Specimen collection and laboratory measurements}

Blood samples were collected between 08:00 and 10:00 hours after 3 days of abstinence from alcohol ingestion and a 12-hour abstinence from smoking and eating. Data on complete blood count, serum electrolytes, homeostatic model assessment of insulin resistance (HOMA1-IR), fasting glucose, lipoprotein fractions (including total cholesterol, high-density cholesterol (HDL) cholesterol, low-density lipoprotein (LDL) cholesterol and serum triglycerides), liver function tests including albumin, GGT, fibrinogen, ferritin and biomarkers like $\mathrm{C}$ reactive protein (CRP), were each determined from appropriately collected and processed samples. Detailed description of the KIHD has been published elsewhere. ${ }^{11}$

\section{Included and excluded subjects}

The initial number of men at baseline was 2682. Of these, we excluded 40 men with history of physician diagnosed liver or pancreas disease, and 162 men with history of diabetes. Of the remaining 2480 men, 1792 who had complete data for FLI calculation, were included in the analyses.

\section{Measuring the components of the FLI}

We calculated FLI using the algorithm developed by Bedogni $e t a l^{7}$ The algorithm incorporates four variables: BMI, waist circumference, serum triglycerides and serum GGT and is expressed as follows:

\section{$\mathrm{FLI}=$}

$$
\begin{aligned}
& \left.\frac{\left(e^{0.953} \times \operatorname{In}(\text { triglycerides })+0.139 \times B M I+0.718 \times \operatorname{In}(g g t)+0.053 \times \text { waist circumference }-15.754\right.}{}\right) \\
& \left(1+e^{0.953 \times I n(\text { triglycerides })+0.139 \times B M I+0.718 \times \operatorname{In}(g g t)+0.053 \times \text { waist circumference }-15.754)}\right. \\
& \times 100
\end{aligned}
$$

where triglycerides is in $\mathrm{mg} / \mathrm{dL}$, waist circumference in $\mathrm{cm}$ and BMI in $\mathrm{kg} / \mathrm{m}^{2}$. We categorised FLI in accordance with Bedogni's categorisation, as low FLI $(<30)$, intermediate FLI $(30-<60)$ and moderate to high FLI $(>60)$, indicating no fatty liver, indeterminate and fatty liver, respectively.

\section{Defining MS status}

MS was defined in accordance with the harmonised criteria for diagnosis of MS. ${ }^{20}$ The presence of any three of the following five risk factors constitutes a diagnosis of MS: waist circumference $\geq 120 \mathrm{~cm}$; serum triglycerides $\geq 150 \mathrm{mg} / \mathrm{dL}(1.7 \mathrm{mmol} / \mathrm{L}) \quad$ or drug treatment for elevated triglycerides); HDL cholesterol $<40 \mathrm{mg}$ / 
$\mathrm{dL}(1.0 \mathrm{mmol} / \mathrm{L})$ (or drug treatment for reduced HDL cholesterol); blood pressure with systolic $\geq 130$ and/or diastolic $\geq 85 \mathrm{~mm} \mathrm{Hg}$ (or antihypertensive drug treatment in a patient with a history of hypertension); and fasting glucose $\geq 100 \mathrm{mg} / \mathrm{dL}$ (or drug treatment of elevated glucose).$^{20}$

\section{Outcome definitions}

We defined incident T2D outcomes as self-reported physician-set diagnosis of T2D and/or fasting plasma glucose $\geq 7.0 \mathrm{mmol} / \mathrm{L}$ or 2-hour oral glucose tolerance test plasma glucose $\geq 11.1 \mathrm{mmol} / \mathrm{L}$ at re-examination rounds 4, 11 and 20 years after the baseline; and T2D information derived by record linkage to either the national hospital discharge registers or to the Social Insurance Institution of Finland register for reimbursement of medicine expenses used for T2D. Detection of T2D by self-report of physician-diagnosed T2D was followed by either detection via the hospital discharge registers or national drug reimbursement register. The proportion of the data obtained by the record linkage are as follows: hospital discharge registers: $42 \%$ and national drug reimbursement register: $58 \%$. T2D cases that were included were those coded in the International Classification of Diseases, 10th Revision (code numbers from E11.0 to E11.9).

\section{Patient and public involvement}

The study was carried out in a non-patient research facility. All the study participants were volunteers. Neither the study participants nor the public were involved in the design of the study.

\section{Statistical methods}

All statistical analyses were performed using SPSS software V.21.0 for Windows. In all analyses, two-sided alpha $<0.05$ was considered statistically significant.

Descriptive analyses were performed to summarise baseline characteristics of participants according to baseline FLI categories. For continuous variables, we used Jonckheere trend test to test for linear trend across FLI categories. For categorical variables, we used $\chi^{2}$ test to test for linear association across FLI categories. To make up for missing $0.4 \%$ values (spread across $50 \%$ of the variables and $13.4 \%$ of subjects), we used a regression-based multiple imputation method (40 iterations) according to guideline by Cheema. ${ }^{21}$

After confirmation of proportionality of hazards, we implemented a multivariable-adjusted Cox proportional hazards model to examine the relationship between baseline FLI and incident T2D considering metabolic factors and the MS statuses of the subjects as follows:

First, we analysed the overall association, adjusting for MS status. The models were as follows: model 1: examination year, constitutional factors (age and family history of T2D), lifestyle factors (smoking pack years, alcohol consumption, physical activity and consumption of fruits, berries and vegetables), inflammatory markers ( $\mathrm{C}$ reactive protein, leucocyte count and thrombocyte count) and metabolic factors (fasting glucose, insulin, HDL, LDL, systolic blood pressure and diastolic blood pressure); model 2: examination year, constitutional factors (age and family history of T2D), lifestyle factors (smoking pack years, alcohol consumption, physical activity and consumption of fruits and berries and vegetables) and inflammatory markers ( $\mathrm{C}$ reactive protein, leucocyte count and thrombocyte count) and MS status.

In sensitivity analyses, we excluded men with a high weekly alcohol consumption of $\geq 168 \mathrm{~g}^{17}$ before analysing the overall association of FLI with T2D in multivariable adjusted Cox proportional hazards as explained above. In addition, we excluded smokers before analysing the overall association of FLI with T2D in multivariable adjusted Cox proportional hazards.

Second, we performed subgroup analyses in which we stratified our study sample by MS status. We then performed multivariable-adjusted Cox proportional hazards analysis with adjustment for covariates to observe if the association of FLI with incident T2D differs by MS status in models 1 and 2 as explained above but excluding fasting glucose in model 2.

Third, for clearer understanding of the relation of the associations considering both FL and MS statuses, using the combination of FLI category and MS status as a composite variable, we performed multivariable-adjusted Cox proportional hazards analysis on the study population with adjustment for covariates as in model 1 above to elaborate the variation of the association by MS status.

\section{RESULTS \\ Characteristics of the study population}

The baseline characteristics of the study population (1792 men) according to FLI categories are shown in table 1. In general, the mean values and proportions for men in the intermediate FLI category were in between estimates for the lowest (reference) FLI category and estimates for the highest FLI category. Compared with the low FLI category, the high FLI category had a greater proportion of men with family history of diabetes and a greater proportion with family history of CVD. Men in the high FLI category consumed less fruit, berries and vegetables and more likely to be heavy alcohol consumers. They had higher mean waist circumference and mean BMI, and they were more likely to be hypertensive. They also had higher GGT levels, higher triglyceride, higher fasting insulin, higher blood glucose, lower HDL cholesterol and higher levels of markers of systemic inflammation. Generally, the range of values of the fasting blood glucose for the eligible men was between $3.1 \mathrm{mmol} / \mathrm{L}$ (minimum) and $6.2 \mathrm{mmol} / \mathrm{L}$ (or $112 \mathrm{mg} / \mathrm{dL}$ ) (maximum).

\section{Multivariable proportional hazards model analyses}

During a mean (SD) follow-up of 18.8 (6.6) years, there were 375 cases of incident T2D. The incidence rate for T2D was 11 cases per 1000 person-years. Significantly lower survival free of incident T2D was noted for participants in high baseline FLI category compared with the low (normal) FLI category 
Table 1 Baseline characteristics of 1792 men according to fatty liver index (FLI) categories

\begin{tabular}{|c|c|c|c|c|}
\hline Characteristic & $\begin{array}{l}\text { FLI }<30 \\
\text { Mean (SD) or } n(\%) \\
n=833\end{array}$ & $\begin{array}{l}\text { FLI }=30-<60 \\
\text { Mean (SD) or } n(\%) \\
n=552\end{array}$ & $\begin{array}{l}\text { FLI } \geq 60 \\
\text { Mean (SD) or } n(\%) \\
n=407\end{array}$ & P trend* \\
\hline FLI & $16.2(7.7)$ & $43.3(8.1)$ & $76.8(10.6)$ & $<0.001$ \\
\hline \multicolumn{5}{|l|}{ Constitutional factors } \\
\hline Age in years & $52.6(5.6)$ & $53.4(5.5)$ & $52.5(5.6)$ & 0.251 \\
\hline Family history of diabetes & $212(25.5 \%)$ & $145(27.8 \%)$ & $108(26.5 \%)$ & 0.651 \\
\hline \multicolumn{5}{|l|}{ Lifestyle factors } \\
\hline Smoking pack years & $7.5(16.0)$ & $8.4(16.8)$ & $6.8(13.6)$ & 0.785 \\
\hline Alcohol consumption (g/week) & $55(89)$ & $78(117)$ & $116(165)$ & $<0.001$ \\
\hline Physical activity (energy exp.) (kcal/day) & 136(156) & $147(175)$ & 129(192) & 0.36 \\
\hline BMI $\left(\mathrm{kg} / \mathrm{m}^{2}\right)$ & $24.4(2.0)$ & $27.3(1.9)$ & $30.7(3.1)$ & $<0.001$ \\
\hline Mean systolic bp & $135.5(17.0)$ & $135.7(17.8)$ & $135.9(18.1)$ & $<0.001$ \\
\hline Mean diastolic bp & $89.4(10.5)$ & $88.5(10.6)$ & $89.6(11.1)$ & $<0.001$ \\
\hline Hypertension & $259(31.1 \%)$ & $275(49.8 \%)$ & $261(64.1 \%)$ & $<0.001$ \\
\hline \multicolumn{5}{|l|}{ Biomarkers } \\
\hline Insulin & $8.3(3.0)$ & $11.2(4.4)$ & $16.6(9.7)$ & $<0.001$ \\
\hline Glucose (mmol/L) & $4.5(0.4)$ & $4.6(0.5)$ & $4.8(0.5)$ & $<0.001$ \\
\hline HOMA1-IR insulin resistance & $1.86(0.71)$ & $2.60(1.10)$ & $3.91(2.30)$ & $<0.001$ \\
\hline Total cholesterol (mmol/L) & $5.71(1.07)$ & $5.93(1.02)$ & $6.05(1.00)$ & $<0.001$ \\
\hline Ferritin $(\mu \mathrm{g} / \mathrm{L})$ & $128(100)$ & $172(157)$ & $235(186)$ & $<0.001$ \\
\hline Fibrinogen $\mathrm{g} / \mathrm{L}$ & $2.92(0.58)$ & $3.06(0.57)$ & $3.10(0.55)$ & $<0.001$ \\
\hline Leucocyte count $\times 10^{9} / \mathrm{L}$ & $5.4(1.6)$ & $5.7(1.6)$ & $5.9(1.6)$ & $<0.001$ \\
\hline \multicolumn{5}{|l|}{ Metabolic syndrome and medication use history } \\
\hline Metabolic syndrome & $29(3.5 \%)$ & $91(16.5 \%)$ & $238(58.5 \%)$ & $<0.001$ \\
\hline Drug for high cholesterol & $7(0.84 \%)$ & $2(0.36 \%)$ & $6(1.47 \%)$ & 0.509 \\
\hline Drug for hypertension & $111(13.32 \%)$ & $127(23.05 \%)$ & $141(34.56 \%)$ & $<0.001$ \\
\hline
\end{tabular}

*Jonckheere trend test for continuous variable. $\chi^{2}$ linear-by-linear association for categorical variables.

BP, blood pressure; CVD, cardiovascular disease; HDL, high-density lipoprotein; HOMA1-IR, homeostatic model assessment of insulin resistance; LDL, low-density lipoprotein.

at baseline (log-rank <0.001). Subjects in intermediate FLI category also separated clearly from those with low FLI for incident T2D.

\section{Relation between baseline FLI and incident T2D}

Overall analyses

Table 2 shows the association of FLI with incident T2D. In model 1, the HRs for incident T2D was $42 \%$ higher for the intermediate category, and $113 \%$ higher for the high FLI category, when compared with the low category. The association was maintained in model 2 with MS where high FLI category was associated with $163 \%$ increased risk. MS was also independently associated with incident T2D in the model with $77 \%$ increased risk (HR (95\% CI): 1.77 (1.35 to 2.31$)$ ).

Sensitivity analyses

After exclusion of 241 men who were heavy alcohol consumers (table 3), the results were similar to those 
Table 2 General association of baseline fatty liver index (FLI) with incident type 2 diabetes

\begin{tabular}{|c|c|c|c|}
\hline FLI & $\begin{array}{l}\text { Number of subjects (\% with } \\
\text { T2D) (IR) }\end{array}$ & $\begin{array}{l}\text { Model } 1 \\
\text { HR }(95 \% \mathrm{Cl})\end{array}$ & $\begin{array}{l}\text { Model } 2 \\
\text { HR }(95 \% \mathrm{CI})\end{array}$ \\
\hline FLI & 1792 (20.9) (11) & $1.013(1.007 \text { to } 1.018)^{*}$ & $1.017(1.012$ to 1.022$) \dagger$ \\
\hline$\leq 30$ (Ref.) & 833 (12.1) (6) & 1.000 & 1.000 \\
\hline $30-<60$ & 552 (22.6) (12) & 1.42 (1.07 to 1.88$)$ & 1.81 (1.38 to 2.37 ) \\
\hline
\end{tabular}

Model 1: FLI, age, examination date, family history of diabetes, smoking pack years, alcohol consumption per week, physical activity, fruit-berry-vegetable consumption, C reactive protein, leucocytes albumin, fibrinogen and ferritin, systolic blood pressure, diastolic blood pressure, insulin, fasting glucose, LDL and HDL.

Model 2: FLI, age, examination date, family history of diabetes, smoking pack years, alcohol consumption per week, physical activity, fruitberry-vegetable consumption, $\mathrm{C}$ reactive protein, leukocytes albumin, fibrinogen and ferritin and metabolic syndrome status.

${ }^{*}$ FLI uncategorised.

†Other independent predictors of T2D in the model were family history of diabetes, serum ferritin, hypertension, serum insulin and glucose. fOther independent predictors of T2D in the model were family history of diabetes, serum ferritin and metabolic syndrome status. HR metabolic syndrome $=1.77(1.35$ to 2.31$)$.

HDL, high-density lipoprotein; IR, incidence rate per 1000 person-years; LDL, low-density lipoprotein; T2D, type 2 diabetes.

obtained in the analyses with the whole sample, as shown in table 2. Similarly, after exclusion of 571 men who were smokers (online supplementary appendix), the results were similar to those obtained in the analyses with the whole sample, as shown in table 1 of the online supplementary appendix.

Further exploration of the association of FLI with incident T2D across FLI categories of 10 (see figure 1) reveals steady increase in HR across the categories without any threshold areas. When we analysed our data with FLI as continuous variable, a unit increase in FLI was associated with $1.7 \%$ increase in HR (in the analyses with the whole sample) and $1.8 \%$ increase (after exclusion heavy alcohol consumers) as shown in tables 2 and 3.

\section{Stratified analyses}

Table 4 shows the results of Cox regression analysis when we stratified by MS status. In the stratus without MS, high FLI was associated with over $100 \%$ increased risk of T2D when compared with those in the low FLI category. Among those with MS, high FLI was not associated with additional risk when compared with those in the low FLI category.

\section{Analysis with composite FLI-MS variable}

In additional sensitivity analyses, with the combination of FLI category and MS status as composite exposure variable, when compared with subjects having neither fatty liver nor MS, having high FLI with no MS was associated with $219 \%$ increase in risk (the HR $(95 \% \mathrm{CI}$ ) was 3.19

Table 3 Association of baseline fatty liver index (FLI) with incident type 2 diabetes after excluding men with high alcohol intake

\begin{tabular}{llll}
\hline FLI & $\begin{array}{l}\text { Number of subjects (\% with } \\
\text { T2D) (IR) }\end{array}$ & $\begin{array}{l}\text { Model 1 } \\
\text { HR (95\% Cl) }\end{array}$ & $\begin{array}{l}\text { Model 2 } \\
\text { HR (95\% Cl) }\end{array}$ \\
\hline FLI* & $1548(20.9)(11)$ & $1.014(1.008$ to 1.019) & $1.018(1.012$ to 1.024) \\
\hline FLI category & & & 1.000 \\
$\leq 30$ (Ref.) & $771(12.5)(6)$ & 1.000 & $1.78(1.33$ to 2.37$)$ \\
$30-<60$ & $472(23.1)(12)$ & $1.43(1.06$ to 1.93$)$ & $2.63(1.89$ to 3.66$)$ \\
$\geq 60$ & $305(38.7)(23)$ & $2.21(1.57$ to 3.10$)$ & $<0.001$ \\
\hline P trend & - & $<0.001$ & \\
\hline
\end{tabular}

${ }^{*} \mathrm{FLI}, \mathrm{FLI}$ uncategorised.

Model 1: FLI, age, examination date, family history of diabetes, smoking pack years, alcohol consumption per week, physical activity, fruit-berry-vegetable consumption, $\mathrm{C}$ reactive protein, leucocytes albumin, fibrinogen and ferritin, systolic blood pressure, diastolic blood pressure, insulin, fasting glucose, LDL and and HDL.

Model 2: FLI, age, examination date, family history of diabetes, smoking pack years, alcohol consumption per week, physical activity, fruitberry-vegetable consumption, $\mathrm{C}$ reactive protein, leucocytes albumin, fibrinogen and ferritin and metabolic syndrome status.

${ }^{*}$ Other independent predictors of T2D in the model were family history of diabetes, serum ferritin, hypertension, serum insulin and fasting glucose.

†Other independent predictors of T2D in the model were family history of diabetes, serum ferritin and metabolic syndrome status. HR metabolic syndrome $=1.65$ (1.24 to 2.21$)$.

HDL, high-density lipoprotein; IR, incidence rate per 1000 person-years; LDL, low-density lipoprotein. 


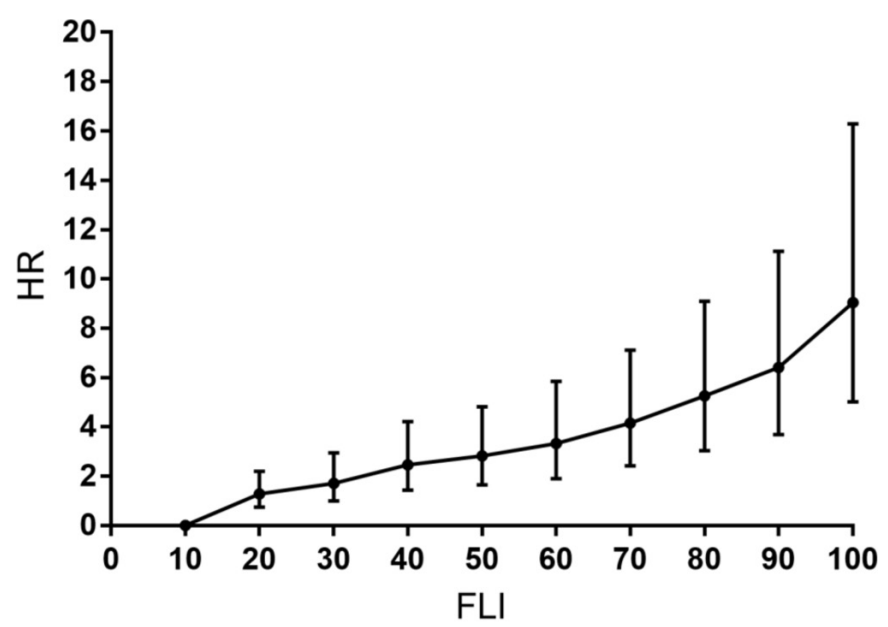

HR= Hazard ratio; FLI= Fatty liver index

Figure 1 Graph of FLI and risk of incident type 2 diabetes.
(2.26 to 4.51)). Having normal FLI with MS was associated with $331 \%$ increased risk (the HR $(95 \% \mathrm{CI}$ ) was 4.31 (2.15 to 8.61)), and persons having high FLI and MS were at greatest risk, with $366 \%$ increase in risk (HR (95\% CI) 4.66 (3.42 to 6.35)). The presence of MS was associated with greater risk in intermediate and high FLI categories (the HRs (95\% CI) were 3.77 (2.50 to 5.70) for presence of MS with intermediate FLI category and 4.66 (3.42 to 6.35) for the presence of MS with high FLI category).

\section{DIABETES PREDICTION \\ Discussion}

We examined the association of FLI, a surrogate of FLD, in relation to incident T2D in a population of middle-aged men while taking the baseline MS status into account. We found that although FLD assessed by FLI predicts the risk

\begin{tabular}{|c|c|c|c|}
\hline No metabolic syndrome & $\begin{array}{l}\text { Number of subjects (\% with } \\
\text { T2D) (IR) }\end{array}$ & Model 1 hour $(95 \% \mathrm{Cl})$ & Model 2 hour $(95 \% \mathrm{Cl})$ \\
\hline $\mathrm{FLI}^{*}$ & 1427 (16.7) (9) & $1.021(1.015$ to 1.027$)$ & $1.017(1.010$ to 1.025$) \dagger$ \\
\hline \multicolumn{4}{|l|}{ FLI category } \\
\hline$\leq 30$ (Ref.) & $803(11.5)(6)$ & 1.00 & $1.00 \ddagger$ \\
\hline $30-<60$ & $456(20.0)(11)$ & 1.81 (1.33 to 2.46$)$ & 1.58 (1.14 to 2.19$)$ \\
\hline$\geq 60$ & 168 (33.3) (18) & 3.07 (2.14 to 4.41$)$ & 2.38 (1.58 to 3.58$)$ \\
\hline $\mathrm{P}$ trend & & $<0.001$ & $<0.001$ \\
\hline \multicolumn{4}{|l|}{ Metabolic syndrome } \\
\hline $\mathrm{FLI}^{*}$ & $358(37.7)(24)$ & 1.007 (0.997 to 1.016$)$ & $0.996(0.992-1.000) \S$ \\
\hline \multicolumn{4}{|l|}{ FLI category } \\
\hline$\leq 30$ (Ref.) & $29(31.0)(23)$ & 1.000 & 1.0009 \\
\hline $30-<60$ & $91(36.3)(21)$ & 0.77 (0.35 to 1.70$)$ & 0.80 (0.59 to 1.09$)$ \\
\hline$\geq 60$ & 238 (39.1) (25) & $1.02(0.49$ to 2.16$)$ & 0.79 (0.58 to 1.06$)$ \\
\hline $\mathrm{P}$ trend & & 0.42 & 0.22 \\
\hline \multicolumn{4}{|c|}{ Category by FLI and MS status } \\
\hline $\mathrm{FLI} \leq 30 \mathrm{MS}$ & $803(11.5)(6)$ & 1.000 & - \\
\hline FLI $30-<60 \mathrm{MS}$ & $456(20.0)(11)$ & 1.79 (1.33 to 2.41$)$ & - \\
\hline $\mathrm{FLI} \geq 60 \mathrm{MS}$ & 168 (33.3) (18) & 3.19 (2.26 to 4.51$)$ & - \\
\hline $\mathrm{FLI} \leq 30 \mathrm{MS}^{+}$ & $29(31.0)(23)$ & 4.31 (2.15 to 8.61$)$ & - \\
\hline FLI $30-<60 \mathrm{MS}^{+}$ & 91 (36.3) (21) & $3.77(2.50$ to 5.70$)$ & - \\
\hline $\mathrm{FLI} \geq 60 \mathrm{MS}^{+}$ & 238 (39.1) (25) & 4.66 (3.42 to 6.35$)$ & - \\
\hline
\end{tabular}

Model 1: FLI, age, examination date, family history of diabetes, smoking pack years, alcohol consumption per week, physical activity, fruitberry-vegetable consumption, $\mathrm{C}$ reactive protein, leukocytes, thrombocytes, fibrinogen and ferritin.

Model 2: Model 1 plus systolic blood pressure, diastolic blood pressure, insulin, LDL and HDL.

${ }^{*}$ FLI uncategorised.IR - incidence rate per 1000 person-years

†Other independent predictors of T2D in the model were serum ferritin and insulin.

¥Other independent predictors of T2D in the model were serum ferritin and insulin.

§Independent predictors of T2D in the model were fasting glucose and insulin.

IIndependent predictors of T2D in the model were fasting glucose and insulin.

Statistically significant at $\mathrm{p} \leq 0.05$.

IR, incidence rate per 1000 person-years; MS, metabolic syndrome; $\mathrm{MS}^{-}$, metabolic syndrome negative; $\mathrm{MS}^{+}$, metabolic syndrome positive; T2D, type 2 diabetes. 
of T2D in the study population, the association was strongest among persons without MS at baseline.

Few studies have investigated the association of baseline FLI as categorised by Bedogni $e t a l$, with incident T2D. ${ }^{102}$ Jäger $e t a l^{22}$ and Onat $e t a l^{10}$ studied the association of FLI with incident T2D in healthy populations, followed up for 8 years. Nishi $e t a l^{23}$ studied the association of FLI with incident T2D in a population of prediabetic subjects followed up for 3 years. ${ }^{23}$

A few studies, Balkau et $a t^{24}$ and Jung et $a l,{ }^{9}$ also reported the association of FLI with incident T2D using FLI categorisation different from that proposed by Bedogni et $a l^{924}$ Because previous studies on the association of FLI with incident T2D have adjusted for different groups of variables in their multivariable models, we are careful in our comparison of findings.

Our finding that high FLI (FLI $\geq 60$ ) indicating fatty liver is associated with increased risk independent of constitutional and lifestyle factors agrees with findings from previous findings by Jäger et $a l^{22}$ and Onat $e t$ al. ${ }^{10}$ We found a 2-3-fold increased risk in our multivariable adjusted models. However, Jäger et al reported 11-fold increase, while Onat et al reported a fivefold increase. Our finding that intermediate FLI is also associated with increased risk of T2D is also in line with reports by Jäger et al.

Our finding that high FLI is associated with incident T2D even after adjusting for metabolic factors agrees with other reports., ${ }^{9} 1024$ each of which used different cut-off points in categorising FLI. Balkau et al used FLI $<20$ and FLI $\geq 70$ as lower and upper cut-off points and adjusted for glucose, insulin and hypertension. Jung et al used FLI $<20$ and FLI $\geq 60$ as lower and upper cut-off points. When we reanalysed our data using these cut-off points, the results (data not shown) did not differ markedly from what we present here.

Indeed, ultrasound diagnosed NAFLD has been shown to be associated with incident T2D, and the association is not affected by adjustment for MS. ${ }^{5}$ However, the predictability of T2D independent of MS using FLI needs to be clarified.

We are unable to compare our findings on association of FLI with incident T2D in view of MS status of the subjects, with previous studies on the association between FLI and incident T2D because previous studies on the association did not consider the MS status of the subjects. However, this finding corroborates the report by Shibata $e t a l^{25}$ Shibata $e t$ al found that the presence of fatty liver, as diagnosed by ultrasonography, is associated with increased risk of T2D when compared with those without fatty liver after adjusting for age, BMI, smoking status, physical activity and MS status. ${ }^{25}$

The finding of similar results, after excluding men who were heavy consumers of alcohol, indicates that our findings are also applicable to NAFLD. However, despite the multifactorial nature of the aetiology of FLD, the relative contribution of heavy ethanol intake in the pathogenesis of fatty liver is still uncertain. ${ }^{26}$ Therefore, we did not exclude men with high alcohol intake in our main analysis. The finding of similar results after excluding smokers proves further that smoking is not a confounder in this target population.

Stratification by MS status did not reveal significant association of high FLI with incident T2D in subjects with MS, despite the fact that increasing proportions of subjects with MS developed T2D across the FLI categories. This suggests that among persons with MS, which is already a cluster of risk factors for T2D (including hyperglycaemia), high FLI is likely not associated with significantly higher risk than that due to positive MS status alone. It also suggests that the MS status was an effect modifier in the overall analysis.

Notwithstanding, our findings from the analysis with FLI-MS composite variable are noteworthy. It appears that FLI predicts risk of T2D in a dose-dependent manner among subjects without MS, but among subjects with MS, it does not predict risk of T2D in a dose-dependent manner. The additional risk associated with high FLI appears less than that associated with MS positive status. Therefore, the greatest risk was in subjects with both fatty liver and MS positivity. Our finding that the presence of MS appears to be associated with higher risks than high FLI may be consistent with the finding by Käräjämäki $e t$ $a l .{ }^{27}$ However, Käräjämäki et al observed from their data that, in the absence of MS, fatty liver does not tend to pose a higher risk for development of T2D in comparison with healthy subjects. ${ }^{27}$ Our finding that, compared with healthy subjects (persons with normal FLI and no MS), persons having high FLI and negative MS status were at increased risk disagrees with their observation.

Comparison of risks with FLI $<10$ as the reference reveals steady increase in risk across FLI (figure 1). This supports the suggestion that, even among subjects with intermediate FLI, the risk of incident T2D increases with increasing FLI values.

Our findings can be explained in the light of current knowledge. It is thought that an initial development of insulin resistance results in compensatory hyperinsulinaemia and, together with visceral obesity, promotes the development of FLD. ${ }^{28}$ In return, the insulin-resistant fatty liver overproduces glucose and very low-density lipoprotein. This boosts mechanisms that lead to exhaustion of pancreatic beta cell reserve, eventually leading to the development of T2D. ${ }^{28}$ Steatotic and inflamed liver secretes hepatokines such as fetuin-A, fetuin-B, angiopoietin-like proteins, fibroblast growth factor 21 and selenoprotein $\mathrm{P}$ that have endocrine function at extrahepatic sites to cause insulin resistance and other adverse effects on glucose homeostasis. ${ }^{29}$ Hence, the association of high FLI with T2D.

Our finding that MS positive status is associated with higher risk than high FLI and the co-occurrence of MS with fatty liver is associated with the greatest risk raises the suspicion that the MS phenomenon represents a more advanced stage than FLD does, in the pathogenesis of T2D, as proposed by Shibata $e t a l^{25}$ and suggested in 
recent epidemiological studies. ${ }^{27}$ However, this does not explain the population of persons with normal liver (low FLI) among people with MS.

The novel finding in our study is that although high FLI (FLD) is associated with increased risk incident T2D, MS phenomenon, which may occur regardless of FLD, modifies this association. However, the association is more clearly demonstrated when the reference group comprises subjects with normal liver and no MS. MS positive status can also predict T2D independent of FLI. In addition, from our data, MS status is associated with higher risk than presence of fatty liver (FLI $\geq 60$ ). However, FLI predicts T2D in subjects without MS. Although FLI appears to be a less efficient predictor of T2D among subjects with MS, the copresence of fatty liver and MS positive status is associated with higher risk than that associated with MS alone. The reason why FLI did not predict T2D among MS subjects is unclear. Our data revealed that among subjects with MS, the association conferred by GGT and BMI (components variables of FLI that are not in included in MS) is not significant when compared with that conferred by insulin resistance and hyperglycaemia. However, this finding of disparate association of FLD with T2D, by MS status, needs to be studied further.

Our current study findings have clinical implications. First, we show that FLI, a surrogate of hepatic steatosis, predicts risk of incident T2D especially in persons who are negative for MS. Second, the association can be affected by metabolic factors or MS status. This suggests that FLD can also play a role in the pathogenesis of T2D. Therefore, both FLD and MS are useful for screening risk of incident T2D. From health systems perspective, because high FLI has also been associated with increased risk of $\mathrm{CVD}^{30}$ and it appears to be detectable before MS may be apparent, screening with FLI may be more cost-effective in asymptomatic persons. The finding of FLI in high category should then prompt further evaluation for T2D and GVD.

Our study does have a number of limitations. First, FLI as a surrogate of fatty liver does not detect progression of FLD. Therefore, we are unable to differentiate the contribution of non-alcoholic steatohepatitis (NASH) and fibrosis to the observed association. Another limitation of the study is that the hepatitis B and hepatitis C statuses of the subjects were not established at baseline. The prevalence rate of hepatitis B and hepatitis $\mathrm{C}$, however, have remained low in the Finnish population. ${ }^{3132}$ Also, our study population comprised of men only. There are reports that suggest that lower FLI cut-off values may apply to women. ${ }^{33}$ We are unable to explore the influence of gender on the predictability of T2D using FLI. Nevertheless, Bedogni $e t a l^{7}$, concluded that the influence of gender in FLI is related to insulin and skinfold thickness and probably insignificant.

The strength of our study lies in the prospective design. With this, we are able to demonstrate the ability of FLI, a surrogate of hepatic steatosis, to predict incident occurrence of T2D. We have also adjusted for a range of constitutional factors, lifestyle factors and biomarkers keeping in cognizance the components of both major exposure variables to control for the possible confounding factors in the predictability of T2D using FLI.

\section{CONCLUSION}

In conclusion, our data show that high FLI category (FLD) is associated with increased risk of incident T2D in men without MS. Persons with high FLI should be further evaluated for FLD, and if FLD is present, they should be evaluated and monitored for T2D. FLD assessed using FLI can be used as additional screening tool for persons at increased risk of incident T2D in the general population. Both FLI and MS are useful and can complement each other in screening and surveillance of persons at increased risk of T2D. In such persons, appropriate preventive or treatment measures should be instituted to improve their prognoses.

Contributors 000 and T-PT conceived the study. 000 and T-PT designed the study. 000 performed the statistical analyses. 000 wrote the first draft of the manuscript. JKV, JP and T-PT contributed towards development of this manuscript. All authors reviewed the final draft of the manuscript.

Funding 000 received study grant from the Finnish Cultural Foundation Central, Finland (Suomen Kulttuurirahasto), in partial support for the conduct of this study. The foundation had no role in the design, conduct or publication of the study. Language editing was by Enembe 0 Okokon, MBBch, MSc, FMCPH, PhD.

Competing interests None declared.

Patient consent for publication Not required.

Ethics approval The study was approved by the Research Ethics Committee of the University of Kuopio, ${ }^{11}$ and the subjects gave their written consent.

Provenance and peer review Not commissioned; externally peer reviewed. Data sharing statement № additional data are available.

Open access This is an open access article distributed in accordance with the Creative Commons Attribution Non Commercial (CC BY-NC 4.0) license, which permits others to distribute, remix, adapt, build upon this work non-commercially, and license their derivative works on different terms, provided the original work is properly cited, appropriate credit is given, any changes made indicated, and the use is non-commercial. See: http://creativecommons.org/licenses/by-nc/4.0/.

\section{REFERENCES}

1. Younossi ZM, Koenig AB, Abdelatif D, et al. Global epidemiology of nonalcoholic fatty liver disease-Meta-analytic assessment of prevalence, incidence, and outcomes. Hepatology 2016;64:73-84.

2. Kwon YM, Oh SW, Hwang SS, et al. Association of nonalcoholic fatty liver disease with components of metabolic syndrome according to body mass index in Korean adults. Am J Gastroenterol 2012;107:107.

3. Chen L, Magliano DJ, Zimmet PZ. The worldwide epidemiology of type 2 diabetes mellitus - present and future perspectives. Nat Rev Endocrinol 2012;8:228-36.

4. Perseghin $\mathrm{G}$. The role of non-alcoholic fatty liver disease in cardiovascular disease. Dig Dis 2010;28:210-3.

5. Musso G, Gambino R, Cassader M, et al. Meta-analysis: natural history of non-alcoholic fatty liver disease (NAFLD) and diagnostic accuracy of non-invasive tests for liver disease severity. Ann Med 2011;43:617-49.

6. Chalasani N, Younossi Z, Lavine JE, et al. The diagnosis and management of non-alcoholic fatty liver disease: practice Guideline by the American Association for the Study of Liver Diseases, American College of Gastroenterology, and the American Gastroenterological Association. Hepatology 2012;55:2005-23. 
7. Bedogni G, Bellentani S, Miglioli L, et al. The Fatty Liver Index: a simple and accurate predictor of hepatic steatosis in the general population. BMC Gastroenterol 2006;6:33.

8. Fedchuk L, Nascimbeni F, Pais R, et al. Performance and limitations of steatosis biomarkers in patients with nonalcoholic fatty liver disease. Aliment Pharmacol Ther 2014;40:1209-22.

9. Jung $\mathrm{CH}$, Lee WJ, Hwang JY, et al. Assessment of the fatty liver index as an indicator of hepatic steatosis for predicting incident diabetes independently of insulin resistance in a Korean population. Diabet Med 2013;30:428-35.

10. Onat A, Can G, Kaya A, et al. Fatty liver disease: Disparate predictive ability for cardiometabolic risk and all-cause mortality. World $\mathrm{J}$ Gastroenterol 2015;21:13555-65.

11. Salonen JT. Is there a continuing need for longitudinal epidemiologic research? The Kuopio Ischaemic Heart Disease Risk Factor Study. Ann Clin Res 1988;20:46-50.

12. Salonen JT, Seppänen K, Nyyssönen K, et al. Intake of mercury from fish, lipid peroxidation, and the risk of myocardial infarction and coronary, cardiovascular, and any death in eastern Finnish men. Circulation 1995;91:645-55

13. Räsänen J, Kauhanen J, Lakka TA, et al. Religious affiliation and allcause mortality: a prospective population study in middle-aged men in eastern Finland. Int J Epidemiol 1996;25:1244-9.

14. Laukkanen JA, Pukkala E, Rauramaa R, et al. Cardiorespiratory fitness, lifestyle factors and cancer risk and mortality in Finnish men. Eur J Cancer 2010;46:355-63.

15. Willenbring ML, Massey SH, Gardner MB. Helping patients who drink too much: an evidence-based guide for primary care clinicians. Am Fam Physician 2009;80:44-50.

16. McGuire S. U.S. Department of Agriculture and U.S. Department of Health and Human Services. Dietary Guidelines for Americans, 2010. 7th Edition. Washington, DC: U.S. Government Printing Office, 2011:293-4.

17. Fawehinmi TO, llomäki J, Voutilainen $\mathrm{S}$, et al. Alcohol consumption and dietary patterns: the FinDrink study. PLoS One 2012;7:e38607.

18. Virtanen JK, Mursu J, Tuomainen TP, et al. Dietary fatty acids and risk of coronary heart disease in men: the Kuopio Ischemic Heart Disease Risk Factor Study. Arterioscler Thromb Vasc Biol 2014;34:2679-87.

19. Kauhanen J, Kaplan GA, Goldberg DE, et al. Pattern of alcohol drinking and progression of atherosclerosis. Arterioscler Thromb Vasc Biol 1999;19:3001-6.

20. Alberti KG, Eckel RH, Grundy SM, et al. Harmonizing the metabolic syndrome: a joint interim statement of the International Diabetes Federation Task Force on Epidemiology and Prevention; National
Heart, Lung, and Blood Institute; American Heart Association; World Heart Federation; International Atherosclerosis Society; and International Association for the Study of Obesity. Circulation 2009;120:1640-5.

21. Cheema JR. Some general guidelines for choosing missing data handling methods in educational research. Journal of Modern Applied Statistical Methods 2014;13:53-75.

22. Jäger S, Jacobs S, Kröger J, et al. Association between the fatty liver index and risk of type 2 diabetes in the EPIC-Potsdam Study. PLoS One 2015;10:e0124749.

23. Nishi T, Babazono A, Maeda T, et al. Evaluation of the fatty liver index as a predictor for the development of diabetes among insurance beneficiaries with prediabetes. J Diabetes Investig 2015;6:309-16.

24. Balkau B, Lange C, Vol S, et al. Nine-year incident diabetes is predicted by fatty liver indices: the French D.E.S.I.R. study. BMC Gastroenterol 2010;10:56.

25. Shibata M, Kihara $Y$, Taguchi M, et al. Nonalcoholic fatty liver disease is a risk factor for type 2 diabetes in middle-aged Japanese men. Diabetes Care 2007;30:2940-4

26. Bedogni G, Bellentani S. Fatty liver: how frequent is it and why? Ann Hepatol 2004:3:63-5.

27. Käräjämäki AJ, Bloigu R, Kauma $\mathrm{H}$, et al. Non-alcoholic fatty liver disease with and without metabolic syndrome: Different long-term outcomes. Metabolism 2017;66:55-63.

28. Valenti L, Bugianesi E, Pajvani U, et al. Nonalcoholic fatty liver disease: cause or consequence of type 2 diabetes? Liver Int 2016;36:1563-79.

29. Targher G, Byrne CD. Clinical Review: Nonalcoholic fatty liver disease: a novel cardiometabolic risk factor for type 2 diabetes and its complications. J Clin Endocrinol Metab 2013;98:483-95.

30. Olubamwo OO, Virtanen JK, Voutilainen A, et al. Association of fatty liver index with the risk of incident cardiovascular disease and acute myocardial infarction. Eur J Gastroenterol Hepatol 2018;30:1047-54.

31. Karvonen T, Auranen K, Kuusi M, et al. Epidemiology of hepatitis B infection in Finland: Implications for immunisation policy. Vaccine 2017;35:412-8

32. Safreed-Harmon K, Hetherington KL, Aleman S, et al. Policy responses to hepatitis $\mathrm{C}$ in the Nordic countries: Gaps and discrepant reporting in the Hep-Nordic study. PLoS One 2018;13:e0190146.

33. Yang BL, Wu WC, Fang KC, et al. External validation of fatty liver index for identifying ultrasonographic fatty liver in a large-scale cross-sectional study in Taiwan. PLoS One 2015;10:e0120443. 